\title{
Dynamic analysis of unilateral diffusion Gompertz model with impulsive control strategy
}

\author{
Yaning $\mathrm{Li}^{1}$, Huidong Cheng ${ }^{1 *}$, Jianmei Wang ${ }^{1}$ and Yanhui Wang ${ }^{1,2}$
}

"Correspondence:

chd900517@sdust.edu.cn

${ }^{1}$ College of Mathematics and

Systems Science, Shandong

University of Science and

Technology, Qingdao, China

Full list of author information is

available at the end of the article

\begin{abstract}
In this paper, we establish a unilateral diffusion Gompertz model of a single population in two patches in a theoretical way. Firstly, we prove the existence and uniqueness of an order-one periodic solution by the geometry theory of differential equations and the method of successor function. Secondly, we prove the stability of the order-one periodic solution by imitating the theory of the limit cycle of an ordinary differential equation. Finally, we verify the theoretical results by numerical simulations.
\end{abstract}

MSC: $34 \mathrm{C} 25 ; 34 \mathrm{D} 20 ; 92 \mathrm{~B} 05 ; 34 \mathrm{~A} 37$

Keywords: unilateral diffusion; order-one periodic solution; limit cycle; Gompertz model

\section{Introduction}

Modern biologists think that the habitat has been deteriorated by an excessive exploitation of resource, and to dodge predators and find suitable habitat, the migration of population becomes necessary [1]. Many researchers focus on this phenomenon and have done a lot of work on it. Skellam [2] investigated the random-walk issue of biological migration and concluded that the balance of the system is related to the size of the habitat. In 1974, Levin and Paine [3] established models of plaque-migratory population dynamics and discussed the influence of the diffusion of population on its survival and extinction. With the development of the research concerned with population diffusion model, more and more people pay attention to diffusion systems. In 1989, Freedman et al. [4] discussed the diffusion system of a single population between two patches and showed that there exists a continuous global asymptotic stable state. In 1994, Zeng et al. [5] focused on the continuous time-diffusion systems and obtained that such a system has a positive and periodic solution.

However, in recent years people have found that the continuous time-diffusion model is not an appropriate description of certain biological phenomena. Some populations spread from one patch to another patch at a fixed time such as the seasonal migration of birds and fish migration. With this in mind, the mathematical model of diffusion with pulse has been established, which makes the research more significant [6-14]. In particular, impulsive differential equations are very important in the research of population migration

(c) The Author(s) 2018. This article is distributed under the terms of the Creative Commons Attribution 4.0 International License (http://creativecommons.org/licenses/by/4.0/), which permits unrestricted use, distribution, and reproduction in any medium, provided you give appropriate credit to the original author(s) and the source, provide a link to the Creative Commons license, and indicate if changes were made. 
phenomenon [15-24]. Jiao et al. [25] established a SIR model with pulse vaccination and proved the existence of a disease-free periodic solution, and a large pulse vaccination rate was a sufficient condition to eradicate the disease. Zou et al. [26] considered a population dynamic system with the delays and impulses and obtained sufficient conditions for the coexistence of two populations. For more applications of differential equations, see [2738].

Recently, Jiao et al. [39] focused on the diffusion predator-prey model of population between two patches and proved that the diffusion rate of the pests and natural enemies in the patch greatly influenced the pest control. Xu et al. [40] established a diffusion model with pulse and proved that the pulse migration was beneficial to the balanced development of the white headed leaf monkey in the fragile environment. Shi et al. [41] built the diffusion system of giant panda in different patches and obtained conditions of pandas' persistence and extinction. However, few researches have been conducted on the dynamics of unilateral diffusion of single population in two patches. Inspired by [39-43], we discuss the following unilateral diffusion Gompertz model [44, 45]:

$$
\left\{\begin{array}{l}
x^{\prime}(t)=a x(t) \ln \left(\frac{K}{x(t)}\right), \\
y^{\prime}(t)=-b y(t), \\
\triangle x(t)=-c x(t), \\
\triangle y(t)=c x(t),
\end{array}\right\} \quad x=\tau,
$$

where $x(t)$ and $y(t)$ indicate the population densities in the two patches at time $t, K$ is the maximum environmental carrying capacity, $a$ is the birth rate of the population, $b$ is the natural mortality of the population within patch two, $0<c<1$ is the migration rate of the population from patch one to patch two, and $0<\tau<K$ is the threshold. In our works, all the parameters are positive.

The paper is organized as follows. In Section 2, we recall some basic results about orderone periodic solutions. In Section 3, we discuss the existence and uniqueness of an orderone periodic solution of system (1) in terms of geometry theory of differential equations and monotonicity of successor function. The stability of an order-one periodic solution is discussed by imitating the theory of the limit cycle of an ordinary differential equation in Section 4. In Section 5, we verify the theoretical results by numerical simulation an make a conclusion.

\section{Preliminaries}

Definition 2.1 ([43]) Consider the following state-dependent impulsive differential functions:

$$
\left\{\begin{array}{l}
x^{\prime}(t)=P(x, y), \\
y^{\prime}(t)=Q(x, y), \\
\triangle x(t)=\xi(x, y), \\
\triangle y(t)=\zeta(x, y),
\end{array}\right\} \quad(x, y) \nsubseteq M_{I}\{x, y\},
$$

The dynamic system composed of a solution mapping defined by system (2) is called a semicontinuous dynamic system, denoted by $\left(\Omega, F, \varphi, M_{I}\right)$, where the function $\varphi$ is a continuous mapping that meets $\varphi\left(M_{I}\right)=M_{I}^{+}$. For convenience, we denote by $M_{I}\{x, y\}$ and 


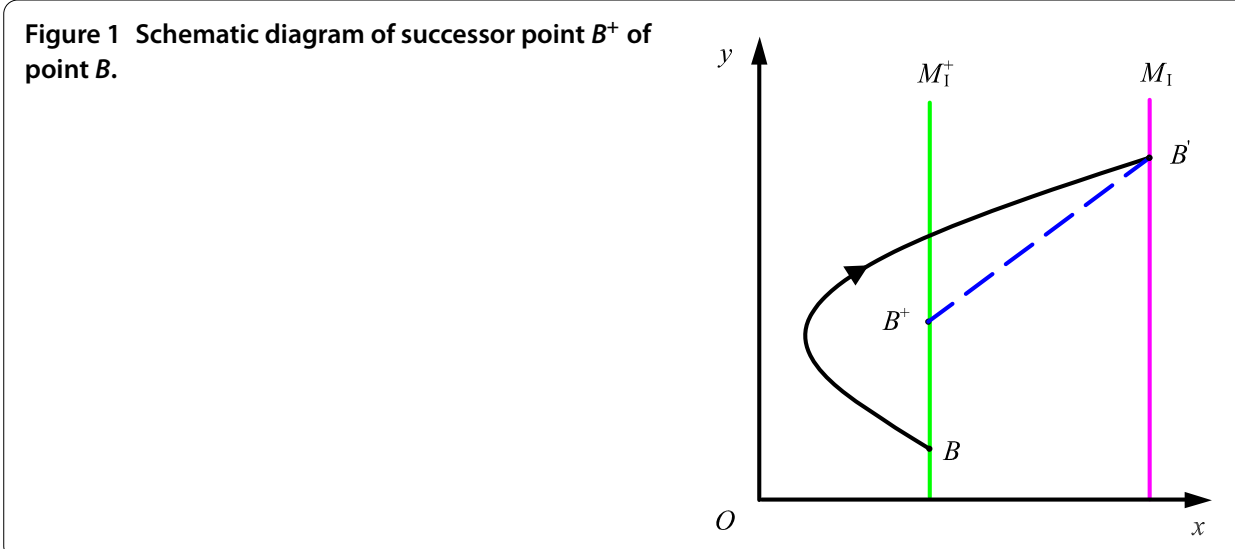

$M_{I}^{+}\{x, y\}$ the straight lines or curves in the plan $R_{2}^{+}=\{(x, y) \mid x \geq 0, y \geq 0\}$, where $M_{I}\{x, y\}$ is called the impulse set, and $M_{I}^{+}\{x, y\}$ is called the phase set.

Remark 2.1 For system (1), we have $M_{I}\{x, y\}=\left\{(x, y) \in R_{2}^{+} \mid x=\tau, y \geq 0\right\}$ and $M_{I}^{+}\{x, y\}=$ $\left\{(x, y) \in R_{2}^{+} \mid x=(1-c) \tau, y \geq 0\right\}$. The impulse mapping is $\varphi:(x, y) \in M_{I} \rightarrow((1-c) \tau$, $\left.c \tau+y_{A}\right) \in R_{2}^{+}$.

Definition 2.2 ([43]) As shown in Figure 1, the trajectory of system (1) beginning from point $B$ intersects with $M_{I}$ at point $B^{\prime}$, then point $B^{\prime}$ jumps to point $B^{+} \in M_{I}^{+}$, and thus the successor function of point $B$ is $f(B)=y_{B}-y_{B^{+}}$.

Lemma 2.1 ([43]) The successor function is continuous.

According to Lemma 2.1, we obtain the following conclusions.

Definition 2.3 ([43]) If there exists a point $B$ of system (1), then we say that the trajectory passing through point $B$ is an order-one periodic solution of system (1) if $f(B)=0$.

Lemma 2.2 ([43]) The system has an order-one periodic solution if there exist two points $C \in M_{I}^{+}$and $D \in M_{I}^{+}$such that the successor function $f(C) f(D)<0$.

Remark 2.2 This lemma is used to prove the existence of order-one periodic solution of system (1) in Section 3.

Lemma 2.3 ([43]) If the successor function of system (1) is a monotonic function, then there exists a unique order-one periodic solution.

Remark 2.3 In Section 3, this lemma is used to prove the uniqueness of order-one periodic solution of system (1).

System (1) with neglected impulse $(c=0)$ is of the form

$$
\left\{\begin{array}{l}
x^{\prime}(t)=a x(t) \ln \left(\frac{K}{x(t)}\right), \\
y^{\prime}(t)=-b y(t) .
\end{array}\right.
$$




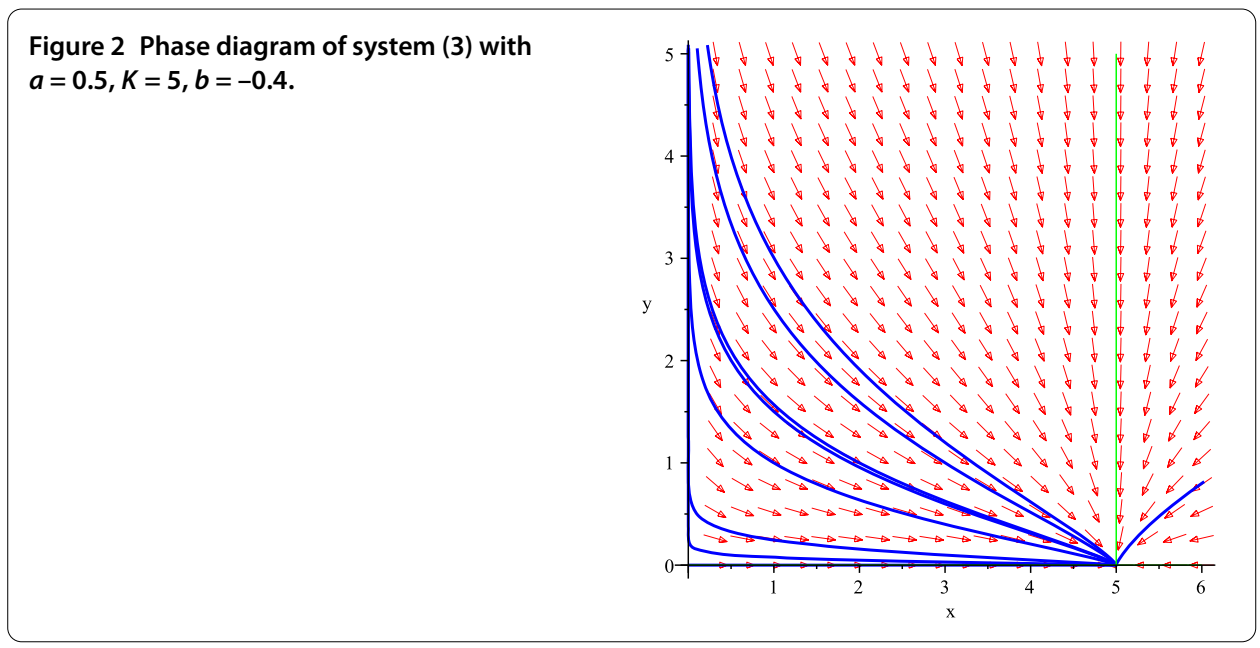

Solving the equations

$$
\left\{\begin{array}{l}
a x(t) \ln \left(\frac{K}{x(t)}\right)=0 \\
-b y(t)=0
\end{array}\right.
$$

we obtain one equilibrium $E^{*}(K, 0)$. Thus, we have the following theorem.

Theorem 2.1 [43] System (3) has a boundary equilibrium, which is a locally stable node.

Proof We discuss the stability of the equilibrium. The Jacobian of system (3) is

$$
J_{0}=\left(\begin{array}{cc}
a \ln \left(\frac{K}{x}\right)-a & 0 \\
0 & -b
\end{array}\right)
$$

At $E^{*}(K, 0)$, we have

$$
J_{E^{*}}=\left(\begin{array}{cc}
-a & 0 \\
0 & -b
\end{array}\right)
$$

with characteristic roots $\lambda_{1}=-a$ and $\lambda_{2}=-b$. Obviously, $E^{*}(K, 0)$ is a locally stable node. The proof is completed.

Furthermore, by Theorem 2.1 we have that $E^{*}(K, 0)$ is locally stable (see Figure 2).

In this paper, we restrict ourselves to the biologically meaningful region $F=\{(x, y) \mid$ $x \geq 0, y \geq 0\}$.

\section{Existence and uniqueness of order-one periodic solution of system (1)}

In this section, we prove the existence and uniqueness of order-one periodic solution of system (1) by geometry theory of differential equations and monotonicity of successor function. As shown in Figure 3, the $x$-isoline $\frac{d x}{d t}=0$ of system (1) intersects with $x$-axis at point $E^{*}(K, 0)$, and the trajectory passing through point $E$ intersects with impulse set $M_{I}$ at point $E^{\prime}(\tau, h)$, and then $E^{\prime}(\tau, h)$ jumps to the phase set at point $E^{+}((1-c) \tau, h+c \tau)$. By 


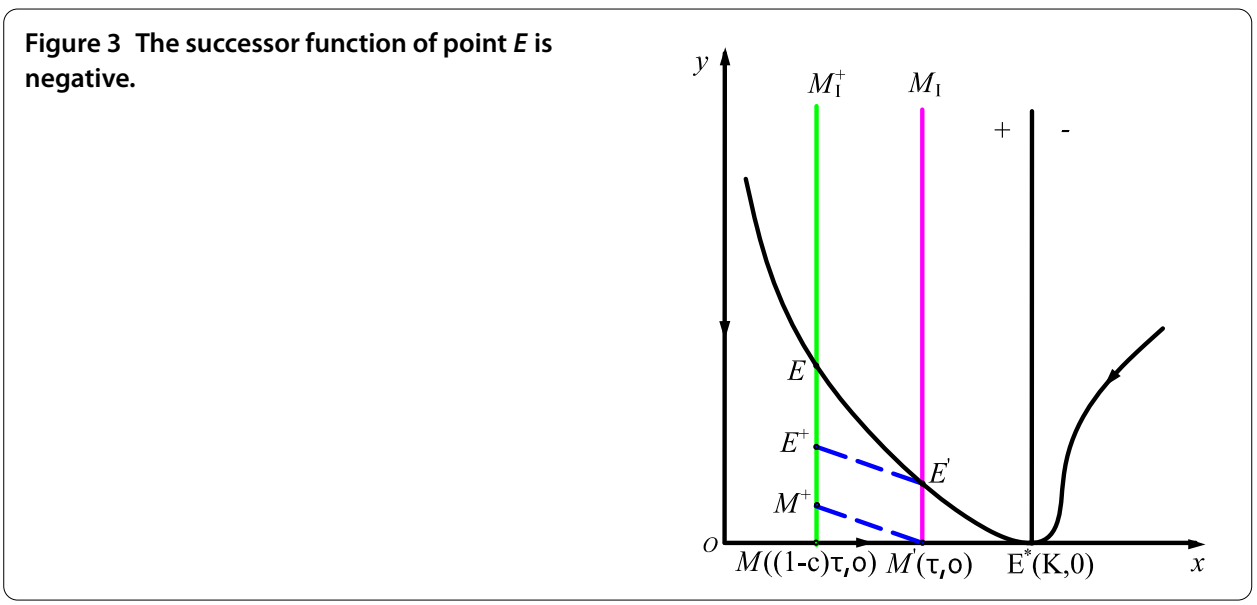

the same method we obtain $M^{+}((1-c) \tau, c \tau)$, which is the successor point of $M$, and the impulse set $M_{I}\{x, y\}$ and the phase set $M_{I}^{+}\{x, y\}$ intersect with $x$-axis at points $M^{\prime}$ and $M$, respectively.

Lemma 3.1 If $h<\frac{a \tau \ln \left(\frac{K}{x}\right)}{b}$, then $k_{E^{\prime} E^{+}}<k_{E^{\prime}}$.

Proof By computing two slopes

$$
\begin{aligned}
& k_{E^{\prime} E^{+}}=\frac{y_{E^{\prime}}-y_{E^{+}}}{x_{E^{\prime}}-x_{E^{+}}}=\frac{c \tau}{(1-c) \tau-\tau}=-1, \\
& k_{E^{\prime}}=\left.\frac{d y}{d x}\right|_{E^{\prime}(\tau, h)}=\left.\left(\frac{d y}{d t} \cdot \frac{d t}{d x}\right)\right|_{E^{\prime}(\tau, h)}=\frac{-b h}{a \tau \ln \left(\frac{K}{\tau}\right)},
\end{aligned}
$$

we get that if $k_{E^{\prime} E^{+}}<k_{E^{\prime}}$, then $-1<\frac{-b h}{a \tau \ln \left(\frac{K}{\tau}\right)}$, The proof is completed.

Remark 3.1 The point $E^{+}$is under the point $E$ when the slope of the trajectory of system (1) at the point $E^{\prime}$ is greater than the slope of the line $E^{\prime} E^{+}$, and so we prove that the successor function of point $E$ is negative.

Lemma 3.2 If $h>\frac{a K}{e c}$, then the straight line $E^{\prime} E^{+}$is not a cutting line of system (1).

Proof The derivative of straight line $E^{\prime} E^{+}: L=y+x-h-\tau$ is

$$
\begin{aligned}
\left.\frac{d L}{d t}\right|_{L=0} & =\left.\left(\frac{d y}{d t}+\frac{d x}{d t}\right)\right|_{t=0} \\
& =\left.\left[-c y+a x \ln \left(\frac{K}{x}\right)\right]\right|_{t=0} \\
& =-c h-c(\tau-x)+a x \ln \left(\frac{K}{x}\right) .
\end{aligned}
$$

Since $\tau>x$, we have

$$
\left.\frac{d L}{d t}\right|_{L=0} \leq-c h+a x \ln \left(\frac{K}{x}\right)<0
$$




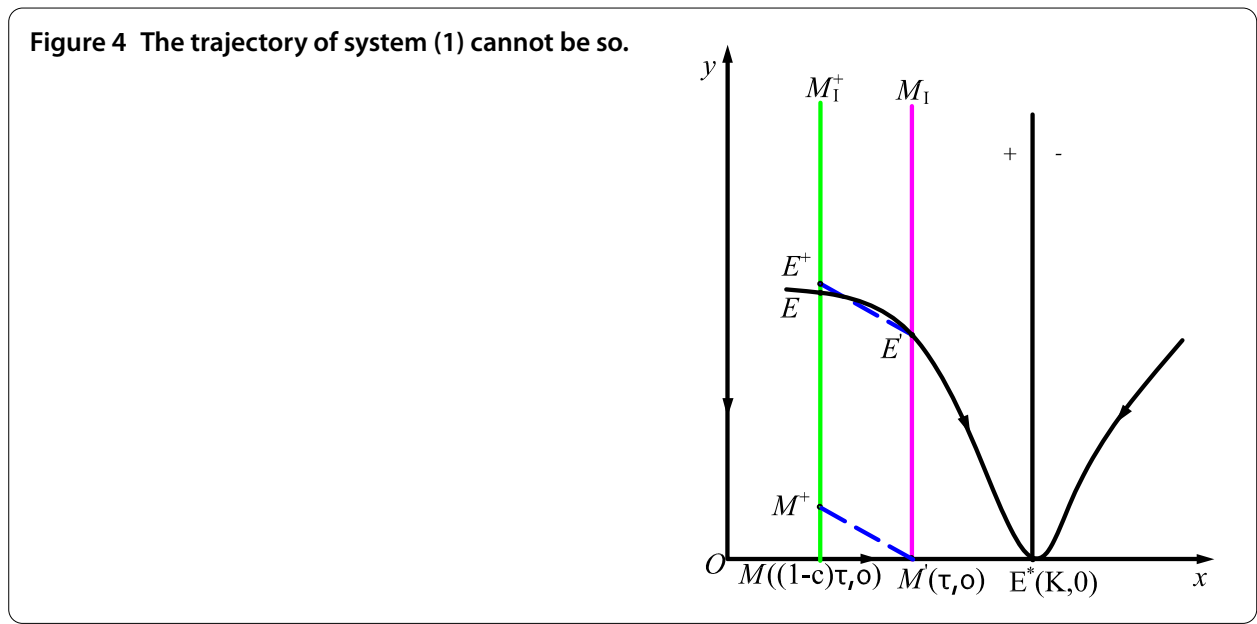

Figure 5 The order-one periodic solution of system

(1).

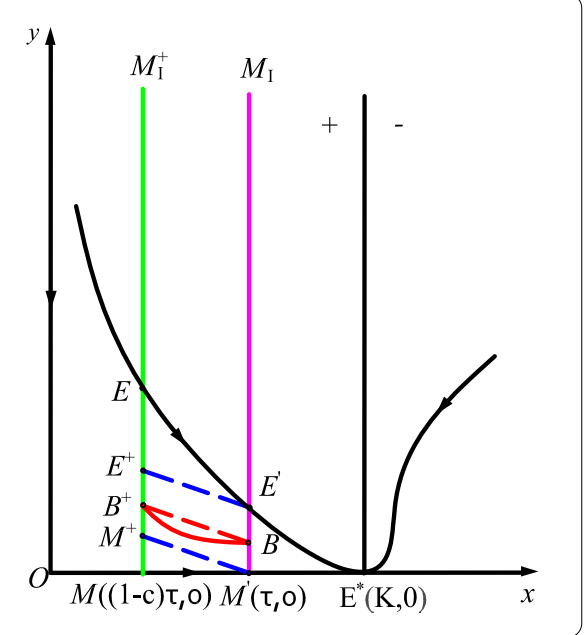

that is,

$$
\operatorname{ax} \ln \left(\frac{K}{x}\right)<c h .
$$

Let $f(x)=a x \ln \left(\frac{K}{x}\right)=0$. Then we get a maximum $x=\frac{K}{e}$. That is to say, if $h>\frac{a K}{e c}$, then $\left.\frac{d L}{d t}\right|_{L=0}<0$, and the trajectory $E E^{\prime}$ of system (1) cannot occur as shown in the situation (see Figure 4). The proof is completed.

Theorem 3.1 System (1) has an order-one periodic solution if $\frac{a K}{e c}<h<\frac{a \tau \ln \left(\frac{K}{\tau}\right)}{b}$ (see Figure 5).

Proof According to Lemmas 3.1 and 3.2, if the point $E^{+}$is under the point $E$, then the condition $\frac{a K}{e c}<h<\frac{a \tau \ln \left(\frac{K}{\tau}\right)}{b}$ is satisfied. The successor function of point $E$ is

$$
f(E)=y_{E^{+}}-y_{E}<0 \text {. }
$$

On the other hand, we choose any point in the pulse set such that the successor function is positive. Based on the condition $\left.\dot{x}\right|_{y=0}>0$, we can choose a point $M((1-c) \tau, 0) \in M_{I}\{x, y\}$ 
and its trajectory passing through the point $M^{\prime}(\tau, 0)$ along a straight line. Then point $M^{\prime}$ jumps to point $M^{+}$owing to impulsive effect, and thus the successor function of point $M$ is

$$
f(M)=y_{M^{+}}-y_{M}=c \tau>0
$$

In summary, according to Lemma 2.2 , in the pulse set $M_{I}^{+}\{x, y\}$, there exists a point $B$ between the points $E$ and $M$ such that $f(B)=0$, that is, system (1) has an order-one periodic solution.

Theorem 3.2 System (1) has a unique order-one periodic solution if $\frac{a K}{e c}<h<\frac{a \tau \ln \left(\frac{K}{\tau}\right)}{b}$ and the successor function of system (1) is monotonic.

Proof In the phase set $M_{I}^{+}$, we choose any two points $F\left(x_{F}, y_{F}\right)$ and $J\left(x_{J}, y_{J}\right)$ between point $M\left(x_{M}, y_{M}\right)$ and point $E\left(x_{E}, y_{E}\right)$ so that the point $J\left(x_{J}, y_{J}\right)$ is under the point $F\left(x_{F}, y_{F}\right)$, and thus their trajectories intersect at points $F^{\prime}\left(x_{F^{\prime}}, y_{F^{\prime}}\right)$ and $J^{\prime}\left(x_{J^{\prime}}, y_{J^{\prime}}\right)$ with impulse set $M_{I}$, respectively, and $y_{F}>y_{J}$ (see Figure 6).

We obtain

$$
y_{F^{\prime}}-y_{F}=\int_{(1-c) \tau}^{\tau} \frac{d y}{d x} d x=\int_{(1-c) \tau}^{\tau} \frac{-b y_{F}}{a x \ln \left(\frac{K}{x}\right)} d x
$$

and

$$
y_{J^{\prime}}-y_{J}=\int_{(1-c) \tau}^{\tau} \frac{d y}{d x} d x=\int_{(1-c) \tau}^{\tau} \frac{-b y_{J}}{a x \ln \left(\frac{K}{x}\right)} d x,
$$

because $y_{F}>y_{J}$. Then

$$
\begin{aligned}
& \int_{(1-c) \tau}^{\tau} \frac{-b y_{F}}{a x \ln \left(\frac{K}{x}\right)} d x<\int_{(1-c) \tau}^{\tau} \frac{-b y_{J}}{a x \ln \left(\frac{K}{x}\right)} d x, \\
& y_{F^{\prime}}-y_{F}<y_{J^{\prime}}-y_{J},
\end{aligned}
$$

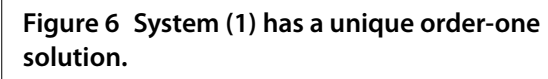


and the successor functions of point $F$ and point $J$ are as follows:

$$
\begin{aligned}
& f(F)=y_{F^{\prime}}-y_{F}, \\
& f(J)=y_{J^{\prime}}-y_{J}, \\
& f(F)-f(J)=\left(y_{F^{\prime}}-y_{F}\right)-\left(y_{J^{\prime}}-y_{J}\right)<0 .
\end{aligned}
$$

Because the successor function of system (1) is monotonic, an order-one periodic solution of system (1) is unique. The proof is completed.

\section{Stability of the order-one periodic solution}

Definition 4.1 ([42] ( $\Omega$ limit set)) On the positive half-trajectory of system denoted by $g\left(p, T^{+}\right), T^{+}=(0,+\infty)$, let $\left\{0 \leq t_{1}<t_{2}<\cdots<t_{n}<\cdots\right\}$ be a time series such that $\lim _{n \rightarrow+\infty} t_{n}=+\infty$. If $P^{*}$ is a limit point of the point range $g\left(p, t_{n}\right), n=1,2, \ldots$, then we say that $P^{*}$ is an $\Omega$ limit point. The set of all $\Omega$ limit points is called the $\Omega$ limit set.

Definition 4.2 ([42]) Suppose $\Gamma^{\prime}$ is an order-one periodic solution of semicontinuous dynamic system. This periodic solution is called stable if it has a sufficiently small neighborhood $\bigcup\left(\Gamma^{\prime}\right)$ such that the $\Omega$ limit set of trajectories starting from any point $Q \in \bigcup\left(\Gamma^{\prime}\right)$ is $\Gamma^{\prime}$.

According to Section 3, we obtain that system (1) has a unique periodic solution. Based on $[42,43]$, using a similar method, we choose any point $A^{\prime} \in M_{I}^{+}$, and the path that consists of the curve $\widehat{A^{\prime} B^{\prime} C^{\prime}}$ and the segment of line $C^{\prime} A^{\prime}$ is an order-one periodic solution, and we denote it by $\Gamma^{\prime}$. Then we choose a point series $\left\{T_{0}^{*}, T_{1}^{*}, \ldots, T_{k}^{*}, T_{k+1}^{*}\right\}$, where $T_{i+1}^{*}$ is the subsequent point of $T_{i}^{*}, i=0,1, \ldots, k, \ldots$. Build the coordinates on $M_{I}^{+}$. The support of the coordinate of $A^{\prime}$ is 0 . Denote by $t_{0}, t_{1}, \ldots, t_{k}, t_{k+1}$ the coordinates of the point series $T_{0}^{*}, T_{1}^{*}, \ldots, T_{k}^{*}, T_{k+1}^{*}$, where $t_{k}=-d_{k}$ when $A^{\prime}$ is on right side of $T_{k}^{*}$, otherwise, $t_{k}=d_{k}$ when $A^{\prime}$ is on left side of $T_{k}^{*}$, and the distance between $T_{k}^{*}$ and $A^{\prime}$ is $d_{k}$ (see Figure 7).

Proposition 4.1 ([43]) For system (1), there exists an order-one periodic solution such that its trajectory is through the point $A^{\prime}$ in the phase set $M_{I}^{+}$. For any point $T_{0}^{*}$ sufficiently close to the point $A^{\prime}$, there exists a point series $T_{0}^{*}, T_{1}^{*}, \ldots, T_{k}^{*}, T_{k+1}^{*}, \ldots \rightarrow A^{\prime}(k \rightarrow \infty)$, that is, $t_{0}, t_{1}, \ldots, t_{k}, t_{k+1}, \ldots \rightarrow 0$, and thus the order-one periodic solution of system (1) is stable. If

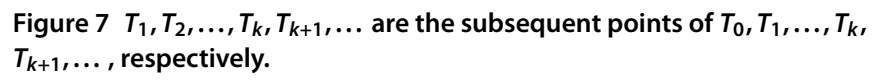


$t_{i}<0\left(t_{i}>0\right)$ for $i=0,1, \ldots, k, \ldots$, then the order-one periodic solution is unidirectionally stable.

Using the result in [43] and a similar method as in [42], we can prove the following results, which will prove the main result of Theorem 4.3. The main idea is to set up a rectangular coordinate to debate the theorems for ascertain the stability of order-one periodic solution.

For system (1), we have a hypothesis that the equations $P(x, y)$ and $Q(x, y)$ have any order partial derivatives, where the partial derivatives of $P$ and $Q$ are denoted by $P_{x_{n}}, P_{y_{n}}, Q_{x_{n}}$, $Q_{y_{n}}$. We assume that the closed orbit $\Gamma^{\prime}$ is an order-one periodic solution of system (1), where $A^{\prime} \in M_{I}^{+}\{x, y\}$ and $C^{\prime} \in M_{I}\{x, y\}$. Suppose that the period from $A^{\prime}$ to $C^{\prime}$ is $T$, so $T$ is also the period of $\Gamma^{\prime}$. In the area very close to $\Gamma^{\prime}$, we build a new coordinate system $(s, n)$. The length of the arc connecting the point $A^{\prime}$ and the corresponding point is the coordinate $s$, and the length of the normal line segment is coordinate $n$, and we suppose downside as the negative direction. Letting $s$ be the parameter, the function of $\widehat{A^{\prime} B^{\prime} C^{\prime}}$ is

$$
\left\{\begin{array}{l}
x=\varphi(s), \\
y=\phi(s) .
\end{array}\right.
$$

Suppose the coordinate of point $A^{\prime}$ is $(\varphi(s), \phi(s))$. Then, let the normal line of trajectory $\Gamma^{\prime}$ pass through $A^{\prime}$, and let it intersect the trajectory starting from $C^{\prime}$ at $D_{k}$. The curve $D_{k}^{\prime} \widehat{E T_{k+1} D_{k+1}^{\prime}}$ will tend to the $\Gamma^{\prime}\left(A^{\prime} B^{\prime} C^{\prime} A^{\prime}\right)$ as $k \rightarrow+\infty$. Then the periodic solution $\Gamma^{\prime}$ is stable (see Figure 8 ). Thus, the relation between the orthogonal coordinate $(s, n)$ and rectangular coordinate $(x, y)$ of point $A^{\prime}$ is

$$
\left\{\begin{array}{l}
x=\varphi(s)-n \phi^{\prime}(s), \\
y=\phi(s)+n \varphi^{\prime}(s),
\end{array}\right.
$$

where

$$
\left\{\begin{array}{l}
\varphi^{\prime}(s)=\left.\frac{d x}{d t}\right|_{A^{\prime}}=\frac{P_{0}}{\sqrt{P_{0}^{2}+Q_{0}^{2}}}, \\
\phi^{\prime}(s)=\left.\frac{d y}{d t}\right|_{A^{\prime}}=\frac{Q_{0}}{\sqrt{P_{0}^{2}+Q_{0}^{2}}},
\end{array}\right.
$$

Figure 8 Establish coordinate system $(s ; n)$ on point $A$.

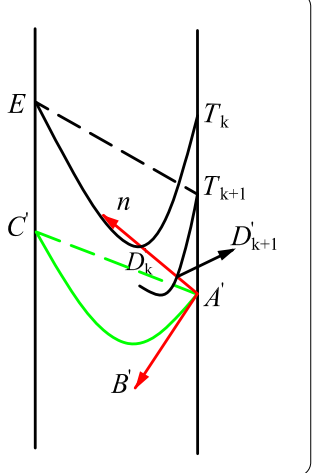


$P_{0}$ and $Q_{0}$ represent the values of the functions $P$ and $Q$ at point $A^{\prime}$, respectively, that is,

$$
\begin{aligned}
& P_{0}=P(\varphi(s), \phi(s)), \\
& Q_{0}=Q(\varphi(s), \phi(s)) .
\end{aligned}
$$

From systems (2) and (5) we have

$$
\frac{d y}{d x}=\frac{\phi^{\prime}(s)+\varphi^{\prime}(s) \frac{d n}{d s}+n \varphi^{\prime \prime}(s)}{\varphi^{\prime}(s)-\phi^{\prime}(s) \frac{d n}{d s}-n \phi^{\prime \prime}(s)}=\frac{Q\left(\varphi(s)-n \phi^{\prime}(s), \phi(s)+n \varphi^{\prime}(s)\right)}{P\left(\varphi(s)-n \phi^{\prime}(s), \phi(s)+n \varphi^{\prime}(s)\right)} .
$$

Then we have

$$
\frac{d n}{d s}=\frac{Q \varphi^{\prime}-P \phi^{\prime}-n\left(P \varphi^{\prime \prime}+Q \phi^{\prime \prime}\right)}{P \varphi^{\prime}+Q \phi^{\prime}} \triangleq F(s, n) .
$$

We know that $n=0$ is a solution of system (8). The function $F(s, n)$ has continuous orderone partial derivative with respect to $n$, and we get

$$
\frac{d n}{d s}=\left.F_{n}^{\prime}(s, n)\right|_{n=0} \cdot n+o(n) .
$$

Because $P_{0} \varphi^{\prime \prime}+Q_{0} \phi^{\prime \prime}=0$, if $n=0$ (see [43]), then we get

$$
\frac{d n}{d s} \triangleq H\left(s^{\prime}\right) n
$$

where $H(x)$ denotes the curvature of orbit at point $A^{\prime}$ for system (2). Therefore the linear approximate function of system (9) is

$$
\frac{d n}{d s}=H(s) n,
$$

and thus the solution is

$$
n=n_{0} e^{\int_{0}^{s} H\left(s^{\prime}\right) d s^{\prime}}
$$

Theorem 4.1 ([43]) If $k$ is the length of arc $\widehat{A^{\prime} B^{\prime} C^{\prime}}$ which is a segment of the one-order periodic solution $\Gamma^{\prime}$ of system (2), then the order-one periodic solution $\Gamma^{\prime}$ is stable (unstable) when

$$
\int_{0}^{k} H(s) d s<0(>0)
$$

Theorem 4.2 ([43]) Assume that the area neared by an order-one periodic solution trajectory $\Gamma^{\prime}$ is convex. If

$$
\int_{0}^{T}\left(P_{x_{0}}+Q_{y_{0}}\right) d t<0
$$

then $\Gamma^{\prime}$ is stable. 
Proof Because $d s=\sqrt{P_{0}^{2}+Q_{0}^{2}} d t$, we have

$$
\begin{aligned}
\int_{0}^{k} H(s) d s & =\int_{0}^{T} \frac{P_{0}^{2} Q_{y_{0}}-P_{0} Q_{0}\left(P_{y_{0}}+Q_{x_{0}}\right)+Q_{0}^{2} Q_{y_{0}}}{P_{0}^{2}+Q_{0}^{2}} d t \\
& =\int_{0}^{T}\left[P_{x_{0}}+Q_{y_{0}}-\frac{P_{0}^{2} P_{x_{0}}+P_{0} Q_{0}\left(P_{y_{0}}+Q_{x_{0}}\right)+Q_{0}^{2} Q_{y_{0}}}{P_{0}^{2}+Q_{0}^{2}}\right] d t \\
& =\int_{0}^{T}\left(P_{x_{0}}+Q_{y_{0}}\right) d t-\frac{1}{2} \int_{0}^{T} \frac{d}{d t}\left[\ln \left(P_{0}^{2}+Q_{0}^{2}\right)\right] d t
\end{aligned}
$$

Because $\Gamma^{\prime}$ is the order-one periodic solution trajectory, we have

$$
\frac{1}{2} \int_{0}^{T} \frac{d}{d t}\left[\ln \left(P_{0}^{2}+Q_{0}^{2}\right)\right] d t=0 .
$$

If

$$
\int_{0}^{T}\left(P_{x_{0}}+Q_{y_{0}}\right) d t<0
$$

then $\int_{0}^{k} H(s)<0$. By Theorem $4.1, \Gamma^{\prime}$ is stable. The proof is completed.

Thus, we get the following conclusions.

Theorem 4.3 ([43]) The order-one periodic solution $\Gamma^{\prime}$ of system (1) is stable if

$$
\int_{0}^{T}\left(\frac{\partial P}{\partial x}+\frac{\partial Q}{\partial y}\right) d t<0
$$

Proof Based on the Bendixson-Dulac theorem (see, e.g., [43]), we can find that system (1) is the topological equivalence system of the following system (12). Let $v(x, y)=\frac{1}{x}$; then

$$
\left\{\begin{array}{l}
\dot{x}=p(x, y) v(x, y)=a \ln \left(\frac{K}{x}\right)=p_{1}(x, y), \\
\dot{y}=q(x, y) v(x, y)=\frac{-b y}{x}=q_{1}(x, y) .
\end{array}\right.
$$

Thus

$$
\frac{\partial p_{1}}{\partial x}+\frac{\partial q_{1}}{\partial y}=-\frac{a}{x}-\frac{b}{x}=\frac{-a-b}{x}<0 .
$$

Therefore the order-one periodic solution of system (1) is stable. The proof is completed.

\section{Numerical simulations and conclusion}

\subsection{Numerical simulations}

In Sections 3 and 4, we have proved that system (1) has a unique stable order-one periodic solution. Here we give an example to verify the results.

Let $a=0.5, K=5, b=0.4, c=0.8$, and let the initial condition be $x_{0}=5, y=2$. By Theorems $3.1,3.2$, and 4.3 system (1) has a unique stable order-one periodic solution. The numerical simulation conforms the result; see Figure 9. 


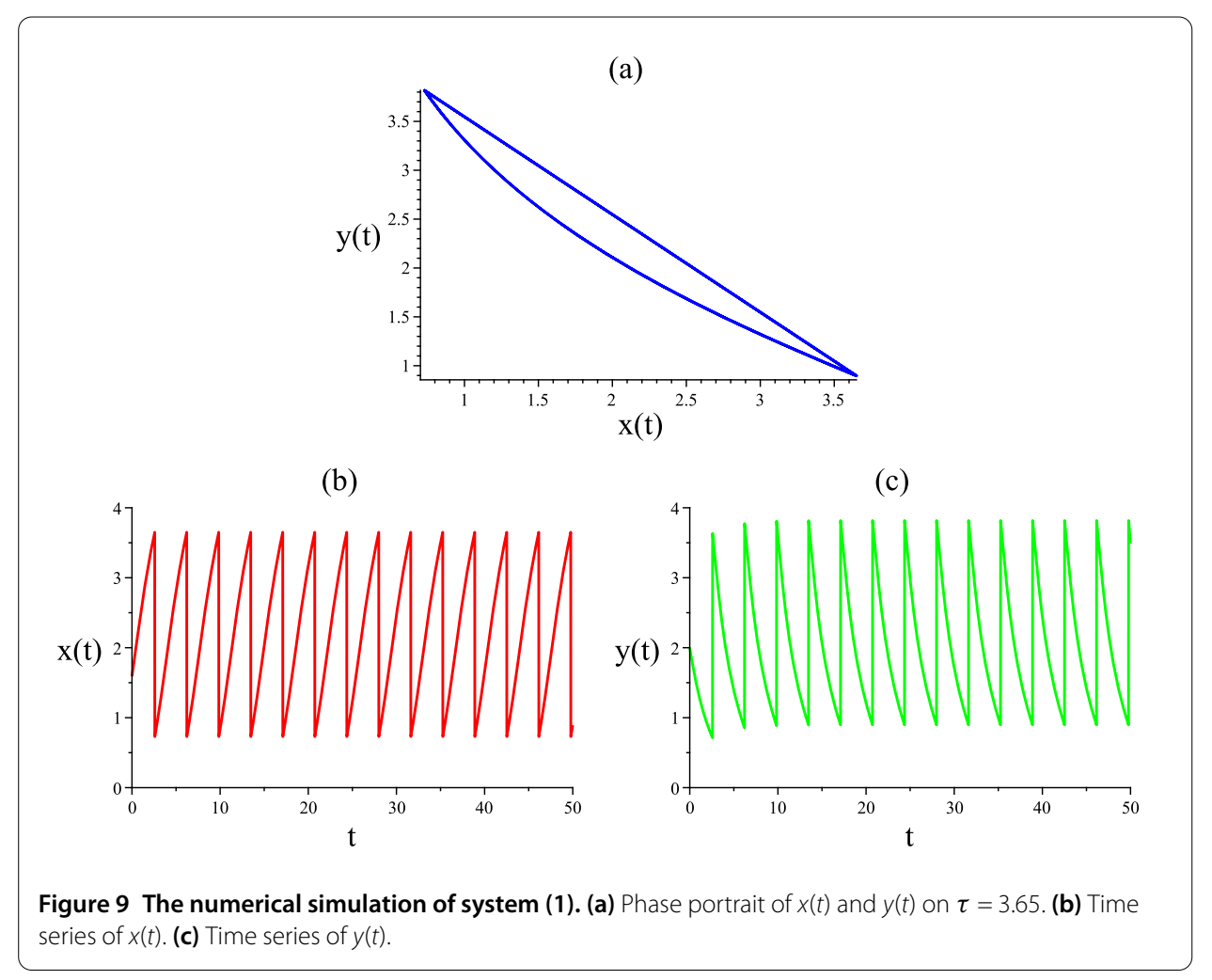

\subsection{Conclusion}

In the previous studies, we always used the Poincaré theorem to prove the stability of order-one periodic solution. However, this method is not suitable for all systems.

In this paper, we compare the slope of pulse line and the trajectory at the pulse point. We get the condition that the successor function is negative, and we prove that system (1) has an order-one periodic solution.

Secondly, we use the monotonicity of the successor function, the geometric theory of differential equations, and the definition of the successor function to prove the uniqueness of an order-one periodic solution.

Finally, we consider a new method to prove the stability of an order-one periodic solution. In Proposition 4.1, the unidirectional stability of an order-one periodic solution can be used to determine that there is no closed orbit around the order-one periodic solution. Using the method of approximating time, we get the same conclusion of the limit cycle theory. So we rewrite Theorem 4.1 to Theorem 4.3. This method solves the problem that the subsequent function is difficult to calculate in the Cartesian coordinate system and also makes the problem simple.

Our main purpose is to prove the existence, uniqueness, and stability of an order-one periodic solution of system (1). However, does there exist a periodic solution for the migration of two- or multipopulation? If it does, then is the periodic solution stable? We leave these for our future work.

\section{Acknowledgements}

The paper was supported by the National Natural Science Foundation of China (No. 11371230, 11501331), Shandong Provincial Natural Science Foundation, China (No. S2015SF002), SDUST Research Fund (2014TDJH102), and Joint 
Innovative Center for Safe and Effective Mining Technology and Equipment of Coal Resources, Shandong Province of China.

\section{Competing interests}

The authors declare that they have no competing interests.

\section{Authors' contributions}

All authors read and approved the final manuscript.

\section{Author details}

${ }^{1}$ College of Mathematics and Systems Science, Shandong University of Science and Technology, Qingdao, China. ${ }^{2}$ State Key Laboratory of Mining Disaster Prevention and Control Co-founded by Shandong Province and the Ministry of Science and Technology, Shandong University of Science and Technology, Qingdao, China.

\section{Publisher's Note}

Springer Nature remains neutral with regard to jurisdictional claims in published maps and institutional affiliations.

Received: 29 September 2017 Accepted: 11 January 2018 Published online: 22 January 2018

\section{References}

1. Andrew Sugden, EP: When to go,where to stop. Science 313, 775 (2006)

2. Skellam, JG: Random dispersal in theoretical populations. Bull. Math. Biol. 53(1), 135-165 (1991)

3. Levin, SA, Paine, RT: Disturbance, patch formation, and community structure. Proc. Natl. Acad. Sci. USA 71(7), 2744-2747 (1974)

4. Freedman, HI, Shukla, JB, Takeuchi, Y: Population diffusion in a two-patch environment. Math. Biosci. 95(1), 111-123 (1989)

5. Zeng, GZ, Chen, LS, Chen, JF: Persistence and periodic orbits for two-species nonautonomous diffusion Lotka-Volterra models. Math. Comput. Model. 20(12), 69-80 (1994)

6. Cheng, $\mathrm{H}$, Zhang, T, Wang, F: Existence and attractiveness of order one periodic solution of a Holling I predator-prey model. Abstr. Appl. Anal. 2012, 1 (2012)

7. Liu, B, Tian, Y, Kang, B: Existence and attractiveness of order one periodic solution of a Holling II predator-prey model with state-dependent impulsive control. Int. J. Biomath. 5(3), 675 (2012)

8. Meng, $X$, Wang, L, Zhang, T: Global dynamics analysis of a nonlinear impulsive stochastic chemostat system in a polluted environment. J. Appl. Anal. Comput. 6(3), 865-875 (2016)

9. Zhang, H, Jiao, J, Chen, L: Pest management through continuous and impulsive control strategies. Biosystems 90 (2), 350-361 (2007)

10. Zhao, W, Li, J, Meng, X: Dynamical analysis of SIR epidemic model with nonlinear pulse vaccination and lifelong immunity. Discrete Dyn. Nat. Soc. 2015, 1-10 (2015)

11. Liu, B, Zhang, Y, Chen, L: Dynamic complexities of a Holling I predator-prey model concerning periodic biological and chemical control. Chaos Solitons Fractals 22(1), 123-134 (2004)

12. Cheng, $H$, Zhang, $T$ : A new predator-prey model with a profitless delay of digestion and impulsive perturbation on the prey. Appl. Math. Comput. 217(22), 9198-9208 (2011)

13. Huang, M, Liu, S, Song, X, Chen, L: Periodic solutions and homoclinic bifurcation of a predator-prey system with two types of harvesting. Nonlinear Dyn. 73, 815-826 (2013)

14. Yang, J, Tang, G, Tang, S: Modelling the regulatory system of a chemostat model with a threshold window. Math. Comput. Simul. 132, 220-235 (2017)

15. Zhang, $T, M a, W$, Meng, $X$, Zhang, T: Periodic solution of a prey-predator model with nonlinear state feedback control. Appl. Math. Comput. 266, 95-107 (2015)

16. Zhang, T, Meng, X, Song, Y, Zhang, T: A stage-structured predator-prey SI model with disease in the prey and impulsive effects. Math. Model. Anal. 18(4), 505-528 (2013)

17. Braverman, E, Liz, E: Global stabilization of periodic orbits using a proportional feedback control with pulses Nonlinear Dyn. 67(4), 2467-2475 (2012)

18. Zhang, T, Ma, W, Meng, X: Global dynamics of a delayed chemostat model with harvest by impulsive flocculant input. Adv. Differ. Equ. 2017, 115 (2017)

19. Zhang, $S$, Meng, $X$, Feng, $T$, Zhang, T: Dynamics analysis and numerical simulations of a stochastic non-autonomous predator-prey system with impulsive effects. Nonlinear Anal. Hybrid Syst. 26, 19-37 (2017)

20. Liu, G, Wang, X, Meng, X: Extinction and persistence in mean of a novel delay impulsive stochastic infected predator-prey system with jumps. Complexity 2017(3), Article ID 1950970 (2017)

21. Zhao, Z, Li, Z, Chen, L: Existence and global stability of periodic solution for impulsive predator-prey model with diffusion and distributed delay. J. Appl. Math. Comput. 33(1-2), 389-410 (2010)

22. Li, Z, Chen, L, Liu, Z: Periodic solution of a chemostat model with variable yield and impulsive state feedback control. Appl. Math. Model. 36(3), 1255-1266 (2012)

23. Tian, Y, Sun, K, Chen, L: Modelling and qualitative analysis of a predator-prey system with state-dependent impulsive effects. Math. Comput. Simul. 82(2), 318-331 (2011)

24. Liu, Q, Huang, L, Chen, L: A pest management model with state feedback control. Adv. Differ. Equ. 2016(1), 292 (2016)

25. Zhou, A, Sattayatham, P, Jiao, J: Dynamics of an SIR epidemic model with stage structure and pulse vaccination. Adv. Differ. Equ. 2016(1), 140 (2016)

26. Zou, L, Xiong, Z, Shu, Z: The dynamics of an eco-epidemic model with distributed time delay and impulsive control strategy. J. Franklin Inst. 348(9), 2332-2349 (2011)

27. Wang, J, Cheng, H, Meng, X, Pradeep, BSA: Geometrical analysis and control optimization of a predator-prey model with multi state-dependent impulse. Adv. Differ. Equ. 2017(1), 252 (2017) 
28. Miao, A, Wang, X, Zhang, T, Wang, W, Sampath Aruna Pradeep, BG: Dynamical analysis of a stochastic SIS epidemic model with nonlinear incidence rate and double epidemic hypothesis. Adv. Differ. Equ. 2017(1), 226 (2017)

29. Tang, S, Chen, L: Global attractivity in a food-limited population model with impulsive effects. J. Math. Anal. Appl. 292(1), 211-221 (2004)

30. Huang, M, Song, X, Li, J: Modelling and analysis of impulsive releases of sterile mosquitoes. J. Biol. Dyn. 11(1), 147-171 (2017)

31. Jiang, G, Lu, Q: Impulsive state feedback control of a predator-prey model. J. Comput. Appl. Math. 200(1), 193-207 (2007)

32. Pang, G, Chen, L: Periodic solution of the system with impulsive state feedback control. Nonlinear Dyn. 78(1), 743-753 (2014)

33. Zhao, L, Chen, L, Zhang, Q: The geometrical analysis of a predator-prey model with two state impulses. Math. Biosci. 238(2), 55-64 (2012)

34. Meng, X, Zhang, L: Evolutionary dynamics in a Lotka-Volterra competition model with impulsive periodic disturbance. Math. Methods Appl. Sci. 39(2), 177-188 (2016)

35. Leng, X, Feng, T, Meng, X: Stochastic inequalities and applications to dynamics analysis of a novel SIVS epidemic model with jumps. J. Inequal. Appl. 2017(1), 138 (2017)

36. Miao, A, Jian, Z, Zhang, T, Pradeep, BGSA: Threshold dynamics of a stochastic SIR model with vertical transmission and vaccination. Comput. Math. Methods Med. 2017, Article ID 4820183 (2017)

37. Liu, L, Meng, X: Optimal harvesting control and dynamics of two-species stochastic model with delays. Adv. Differ. Equ. 2017(1), 18 (2017)

38. Jiang, Z, Zhang, T: Dynamical analysis of a reaction-diffusion phytoplankton-zooplankton system with delay. Chaos Solitons Fractals 104, 693-704 (2017)

39. Jiao, J, Yang, X, Cai, S, Chen, L: Dynamical analysis of a delayed predator-prey model with impulsive diffusion between two patches. Math. Comput. Simul. 80(3), 522-532 (2009)

40. Xu, W, Chen, L, Chen, S, Pang, G: An impulsive state feedback control model for releasing white-headed langurs in captive to the wild. Commun. Nonlinear Sci. Numer. Simul. 34, 199-209 (2016)

41. Shi, X, Yang, J, Song, G: Study on a giant panda reintroduction state feedback control pulse model with diffusion between two patches. J. Appl. Math. Comput. 51(1), 271-285 (2016)

42. Zhang, M, Song, G, Chen, L: A state feedback impulse model for computer worm control. Nonlinear Dyn. 85(3), 1561-1569 (2016)

43. Sun, M, Liu, Y, Liu, S, Hu, Z, Chen, L: A novel method for analyzing the stability of periodic solution of impulsive state feedback model. Appl. Math. Comput. 273, 425-434 (2016)

44. Zhang, T, Meng, X, Liu, R, Zhang, T: Periodic solution of a pest management Gompertz model with impulsive state feedback control. Nonlinear Dyn. 78(2), 921-938 (2014)

45. Trzcinski, MK, Reid, ML: Intrinsic and extrinsic determinants of mountain pine beetle population growth. Agric. For. Entomol. 11(2), 185-196 (2009)

\section{Submit your manuscript to a SpringerOpen ${ }^{\circ}$ journal and benefit from:}

- Convenient online submission

- Rigorous peer review

- Open access: articles freely available online

- High visibility within the field

- Retaining the copyright to your article

Submit your next manuscript at $>$ springeropen.com 